\title{
Band Based Power Control (BBPC) for MC-CDMA Radio Interface
}

\author{
Asif Hamid, Reza Hoshyar, Rahim Tafazolli \\ Center for Communication Systems Research \\ University of Surrey \\ Guildford (GU2 7UZ), UK \\ A.Hamid@surrey.ac.uk
}

\begin{abstract}
This paper highlights, limitations of ECPC (Each Carrier Power Control) concept [1] - originally proposed for OFDM-DS-CDMA - when extended to MC-CDMA (MultiCarrier Code Division Multiple Access) systems. First, its impractical signaling overhead of $80 \%$ secondly; its inability to be used as an uplink power control mechanism. Then we propose BBPC (Band Based Power Control) as a practical alternative to ECPC for MC-CDMA systems. Unlike, ECPC that controls power on each carrier basis, BBPC assigns same power level to a band of carriers (lying within coherence bandwidth of channel). It has been shown that with a nominal performance loss, BBPC reduces the signaling overheads to $2.5 \%$ and by employing the control index estimator after de-spreading, it can be used as an uplink power control mechanism for MC-CDMA. We have used SIR (Signal to Interference Ratio) as a power control index, BER and standard deviation of power control error as performance metrics.
\end{abstract}

Keywords; power control; uplink; MC-CDMA; coherence bandwidth.

\section{INTRODUCTION}

Recently, MC-CDMA (Multi-Carrier Code Division Multiple Access) has gained significant interest as a multiple access method and has emerged as a potential candidate for $4 \mathrm{G}$ cellular systems [2-3]. This is mainly due to its capability of simultaneously exploiting the advantages of OFDM (Orthogonal Frequency Division Multiplexing) and CDMA (Code Division Multiple Access) to cope with frequency selective fading encountered in large bandwidths and thereby ensuring very high data rates required for $4 \mathrm{G}$ systems. Being CDMA based system; MC-CDMA is susceptible to MAI (Multiple Access Interference) [6]. Apart from MAI, near far effect [6] and fast fading experienced by the signal, causes deterioration in received power, resulting in performance loss. To compensate these effects while maintaining reasonable Quality of Service (QoS), a fast and accurate mechanism of controlling the power of transmitted signal is required for $\mathrm{MC}$ CDMA [4-5].

Opposed to single carrier CDMA systems, a more comprehensive power control scheme is required for $\mathrm{MC}$ CDMA, because data is transmitted over several orthogonal sub-carriers in this system. Furthermore, depending upon the coherence bandwidth of channel the received power level on different sub-carrier may results in appreciably different

Asif's work was supported by a scholarship award from NCP (Quaid-I-Azam) University Islamabad Pkaistan. values. Hence, for a precise control over transmitter power, ideally, power assignment must be done on carrier-to-carrier basis that pose many problems (i.g. feedback bandwidth requirement for transmission of control bits) not encountered in single carrier systems.

Although, there has been a significant amount of work [6] reported into the investigation of TPC (Transmit Power Control) techniques for single carrier systems like W-CDMA, however very few attempts $[1,4,5,7]$ have been reported for multi-carrier systems so far. Works reported in [1] and [4] target OFDM-DS-CDMA systems only and don't encompass MC-CDMA, whereas [5] and [7] only establish a requirement for power control in MC-CDMA systems rather than proposing any practicable TPC technique for it. ECPC (Each Carrier Power Control) has been shown [1] to outperform ACPC (All Carrier Power Control), but concept of ECPC can't be extended to the system under consideration (i.e. MC-CDMA) mainly due to its following limitations:

- $\quad$ ECPC is meant to control power on per sub-carrier basis, so control index (power or SIR) needs to be estimated prior to combining operation [Fig. 2]. Since, in MC-CDMA, spreading is done in frequency domain using user specific codes [Fig. 1], therefore desired user data can't be segregated prior to de-spreading. Hence idea of ECPC can't be extended to MC-CDMA environment.

- $\quad$ Being uplink power control mechanism, ECPC needs to be implemented in closed loop [6], hence, requires a certain feedback bandwidth on downlink. With reference to simulation parameters [Table II], a signaling overhead analysis of ECPC has been carried out [Table I], which resulted in an unfeasibly high feedback overhead of $80 \%$, so ECPC is not even a suitable TPC mechanism for MC-CDMA systems.

Therefore keeping in mind the limitations of ECPC, this paper proposes a Band Based Power Control (BBPC) mechanism that exploits the coherence bandwidth information $B_{c}$ of channel, and controls the power of a band of sub-carriers (lying within $B_{c}$, hence undergoing a highly correlated fading process) with single power control command. We demonstrate through simulations that against a nominal performance loss of less than $.5 \mathrm{~dB}, \mathrm{BBPC}$ remedies both of the above stated 
limitations of ECPC that makes it a suitable and practically realizable TPC mechanism for MC-CDMA radio interface.

Rest of this paper has been organized as follows; in section II we describe the system's conceptual model used in this study, section III elaborates the BBPC concept. Comparison of BBPC performance against ECPC and ACPC is given in section IV using simulation results. This section also validates the concept of BBPC using channels of different coherence bandwidths and also by varying the bandwidth occupied by one band of sub-carriers, whereas section V concludes the paper.

TABLE I. SIGNALLING OVERHEAD ANALYSIS OF ECPC

\begin{tabular}{|c|c|c|c|}
\hline No & Parameter & Symbol & Value \\
\hline 1 & Baseband modulation index & $\mathrm{m}$ & 2(QPSK) \\
\hline 2 & No. of parallel branches & $\mathrm{P}$ & 23 \\
\hline 3 & Information bits per OFDM symbol & $\mathrm{I}=\mathrm{m} * \mathrm{P}$ & 46 \\
\hline 4 & Frame length [OFDM symbols] & Ns & 20 \\
\hline 5 & No. of Information bits per frame & $\mathrm{I}_{\mathrm{b}}=\mathrm{I}^{*} \mathrm{Ns}$ & 920 \\
\hline 6 & Spreading factor & $\mathrm{K}_{\mathrm{MC}}$ & 32 \\
\hline 7 & Number of data sub-carriers & $\begin{array}{c}\mathrm{Nsc}= \\
\mathrm{K}_{\mathrm{MC}} * \mathrm{P}\end{array}$ & 736 \\
\hline 8 & Required control bits per frame & $\mathrm{C}_{\mathrm{b}}=\mathrm{Nsc}$ & 736 \\
\hline 9 & $\begin{array}{l}\text { Signalling overhead (fixed step } \\
\text { mode) }\end{array}$ & $\mathrm{C}_{\mathrm{b}} / \mathrm{I}_{\mathrm{b}}$ & $80 \%$ \\
\hline
\end{tabular}

\section{SySTEM ARChiteCtURE}

\section{A. Transceiverr Model}

The transmitter part of MC-CDMA for $j^{\text {th }}$ user is modeled as shown in Fig. 1. Random binary data stream is generated with bit duration of $T_{b}$, and passed through a QPSK modulator. This serial stream of modulated symbols is converted to $P$ parallel streams $a_{j, p}[p=0,1, \ldots . P-1]$ thereby increasing symbol duration to $T_{s}=T_{b} / P$. Each of these streams is then subjected to spreading in frequency domain. This is where CDMA feature has been incorporated; user specific codes (Walsh Hadamard) $d_{j, m}\left[m=0,1, \ldots . K_{M C}-1\right]$ with spreading factor of $K_{M C}$ have been used. This spreaded signal consists of $P^{*} K_{M C}$ chips each with duration of $T_{s}$. Prior to multi-carrier modulation, to avoid burst errors these chips are interleaved over different frequency carriers in Frequency Interleaving block. Finally all of these streams are applied to the multi-carrier modulation using IFFT to generate OFDM symbols. However, to avoid ISI (Inter Symbol Interference), prior to transmission a cyclically extended guard interval of duration $\tau_{g}$ (depending upon the channel delay spread) is inserted before every OFDM symbol.

On receiving end [Fig. 2], the received signal $r(t)$ is serial to parallel converted, cyclic prefix is removed and multi-carrier demodulation is performed using FFT. Prior to combining, frequency de-interleaving is done. Channel Estimator provides an estimation of channel response $Z_{j, p, m}$, which denotes the channel complex gain of the $(m P+p)^{t h}$ sub-carrier of the $j^{\text {th }}$ user. Based on combining technique, the combiner coefficients $G_{j, p, m}$ are calculated. Equation (1) gives these coefficients for Equal Gain Combining (EGC) [9].

$$
G_{j, p, m}=\frac{d_{j, m} Z_{j, p, m}^{*}}{\left|Z_{j, p, m}\right|}
$$

These coefficients are then supplied to the combiner that combines energy of the received signal scattered in frequency domain. The decision variable for $j^{\text {th }}$ user and $p^{\text {th }}$ parallel stream $D_{j, p}$, is obtained as given by (2).

$$
D_{j, p}(i)=\sum_{m=0}^{K_{M C}-1} G_{j, p, m}(i) Y_{p, m}(i)
$$

Where $Y_{p, m}(i)$ is received, demodulated and frequency deinterleaved signal on $(m P+p)^{\text {th }}$ sub-carrier as given by (3).

$$
Y_{p, m}(i)=\sum_{j=0}^{J-1} Z_{j, p, m}(i) a_{j, p} d_{j, m}+n_{p, m}(i) \ldots
$$

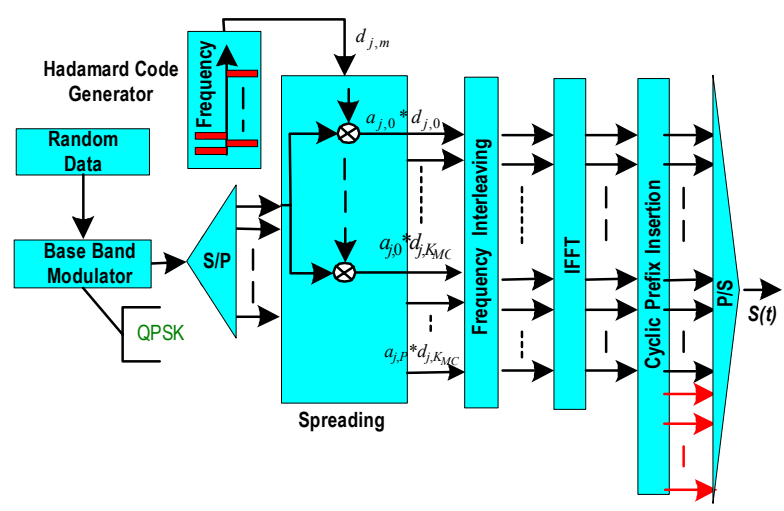

Figure 1. MC-CDMA Transmitter Architecture

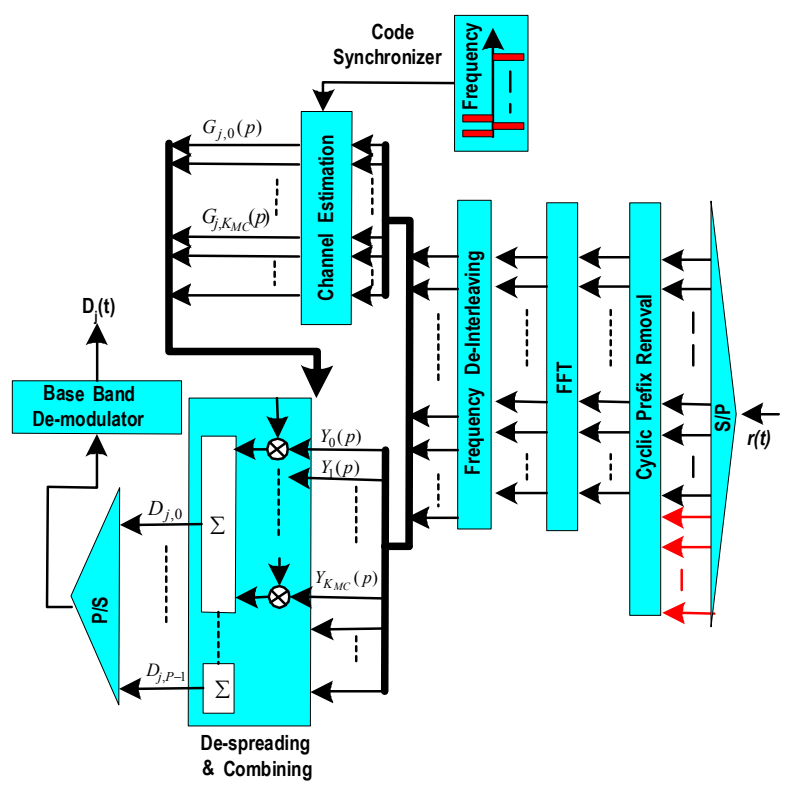

Figure 2. MC-CDMA Receiver Architecture 


\section{B. Multipath Channel Model}

Multi-path Rayleigh channel is simulated using Jakes model of sum of sinusoids [8] and is characterized by its PDP (Power Delay Profile). We have employed PDP given by Table II. Parameter $K_{M C}$ in this table is chosen so that the value $\Delta f^{*} K_{M C}$ becomes smaller than the coherence bandwidth $B_{c}$ of the frequency selective channel (the reason for this assumption will become clear in the sequel). Where $\Delta f$ is inter-carrier spacing given by (4).

$$
\Delta f=\frac{1}{T_{s}-\tau_{g}}
$$

\section{BAND BASED POWER CONTROL}

As described in section II-A, data on each parallel stream is spread in frequency domain. Furthermore, in absence of frequency interleaving; it is transmitted on adjacent carriers. Therefore, in absence of any frequency interleaving and by subjecting the spreading factor $K_{M C}$ and $\Delta f$ to (5) we can ensure highly correlated fading on all $K_{M C}$ number of sub-carrier being modulated by a single data stream $a_{j, p}$.

$$
K_{M C} \Delta f \leq B_{c}
$$

Therefore, one single power control command is theoretically justified for all of these $K_{M C}$ adjacent sub-carriers lying within coherence bandwidth of channel. Thereby, reducing the feedback bandwidth by a factor of $K_{M C}$ (as compared to ECPC) ideally without any loss of BER performance. Furthermore, by measuring the power control index after de-spreading, the second limitation of ECPC can also be eliminated, which is the main theme followed by BBPC. Fig.3 shows BBPC in MC-CDMA transmission loop. We have used it in adaptive step mode which uses multi-level power control commands $P_{c m} d_{j, p}$ for each user and each band of sub-carriers generated by Power Controller as given by (6)

$$
\text { Pcmd }_{j, p}(k)=\left\lfloor\frac{S \operatorname{SR}_{r, j, p}(k)-\operatorname{SIR}_{t g, j, p}(k)}{\text { stepres }}\right\rfloor \ldots \ldots \ldots \ldots(6)
$$

Where $S I R_{r, j, p}[10]^{\text {and }} S I R_{t g, j, p}$ are received and target SIRs of $j^{\text {th }}$ user on $p^{\text {th }}$ band (stream). These TPC commands accordingly adjust the transmit power of $P_{T, j, p}(k)$ of $j^{t h}$ user and $p^{\text {th }}$ band in the $k^{\text {th }}$ frame by an integral multiple of a minimum power excursion step size stepres nearest to the actual difference of received and target value of control index as given by (7).

$$
P_{T, j, p}(k)=P_{T, j, p}(k-1) 10{\frac{P_{\text {cand }, j, p}{ }^{*} \text { stepres }}{20}}^{2 \ldots \ldots . .(7)}
$$

\section{Simulations AND RESUltS}

The BBPC concept described in previous section is validated through numerous system level simulations targeting different aspects like its performance against ECPC and ACPC and its performance for different channel coherence bandwidths (i.e. delay spread). BER and standard deviation of PCE (Power Control Error) are chosen to be the performance metrics for this comparison. We assume one OFDM symbol delay $\left(\tau_{d}=1\right)$ in generation of power control commands by Power Controller and their implementation at Power Amplifier [Fig. 3]. All of the simulation parameters assume the values tabulated in Table-II unless specified differently.

First of all the BER performance of BBPC was investigated as function of target SIR [Fig. 4] at a constant channel fading rate characterized by mobile's normalized speed $\left(F_{d} T_{p}\right)$ of 0.0598 that corresponds to an average mobile speed of 30 $\mathrm{Km} / \mathrm{hr}$ and power control period of $0.43 \mathrm{~ms}$. Fig. 3 shows that there is hardly any performance difference between BBPC and ECPC, however, performance difference between ACPC and BBPC kept on increasing with increase in SIR target, which is attributed to the decreasing interference from AWGN at higher values of target SIR.

Since for a TPC validation, its channel tracking ability is a key metric, so it becomes essential to study its performance as function of channel fading rate as well. This feature of BBPC is investigated and compared against ECPC and ACPC in Fig. 5. The study reveals that although BER performance of ECPC and BBPC is nearly identical, but ECPC outperforms its other two alternatives as far as its channel tracking ability is concerned. It can also be seen from Fig. 5 that standard deviation of PCE for all three TPC techniques are appreciably different at low channel fading rates experienced by pedestrians $\quad\left(F_{d} T_{p}=0.00688\right)$. However, this performance difference diminishes with increase in fading rates and

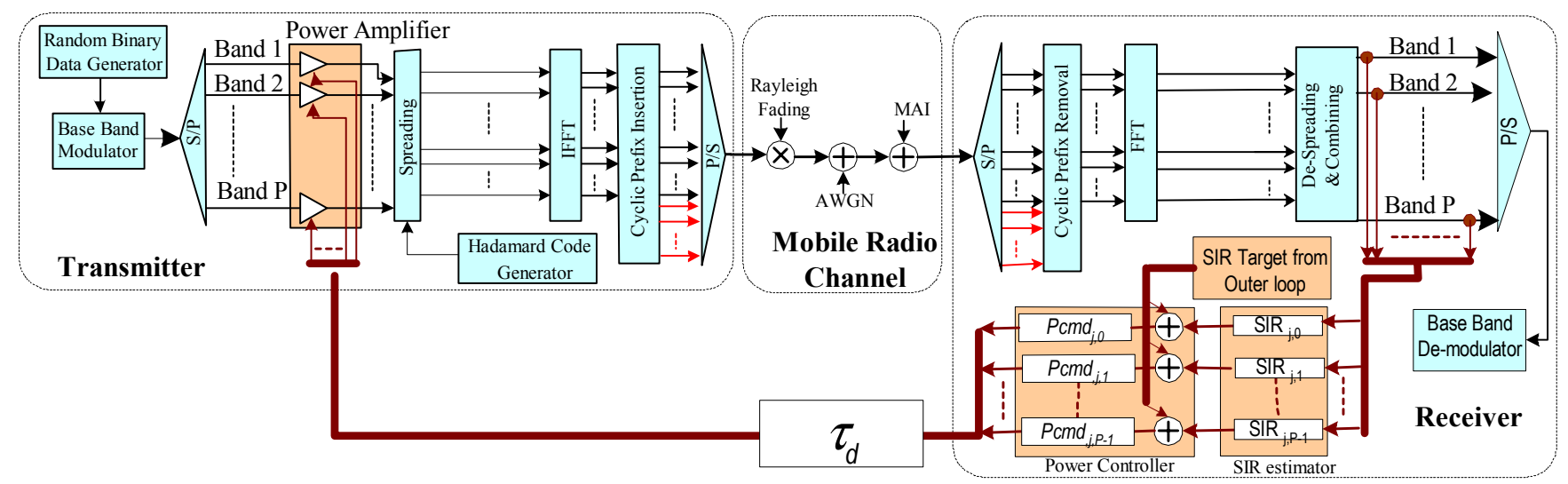

Figure 3. BBPC in MC-CDMA transmission loop 
ultimately all three curves converge to a single asymptotic value of $4.5 \mathrm{~dB}$ at $F_{d} T_{p}=0.2$. However, BBPC competes ECPC well with a nominal performance loss of $0.27 \mathrm{~dB}$ at $F_{d} T_{p}=0.0598$.

\section{TABLE II. SIMULATION PARAMETERS}

\begin{tabular}{|c|c|c|c|}
\hline No & Parameter & Symbol & Value \\
\hline 1. & RF carrier frequency & $\mathrm{F}_{\mathrm{c}}$ & $5 \mathrm{GHz}$ \\
\hline 2. & Baseband modulation index & $\mathrm{m}$ & 2(QPSK) \\
\hline 3. & No. of parallel branches & $P$ & 23 \\
\hline 4. & Spreading Codes & \multicolumn{2}{|c|}{ Hadamard Walsh } \\
\hline 5. & Spreading factor & $\mathrm{K}_{\mathrm{MC}}$ & 32 \\
\hline 6. & FFT size & $\mathrm{N}_{\mathrm{FFT}}$ & 1024 \\
\hline 7. & Useful symbol duration & $\mathrm{T}_{\mathrm{b}}$ & $17.75 \mu \mathrm{s}$ \\
\hline 8. & Guard Interval & $\tau_{g}$ & $3.75 \mu \mathrm{s}$ \\
\hline 9. & Symbole total duration & $T_{s}$ & $21.506 \mu \mathrm{s}$ \\
\hline 10. & Sampling frequency & $f_{s}$ & $57.6 \mathrm{MHz}$ \\
\hline 11. & Combining technique & \multicolumn{2}{|c|}{ EGC } \\
\hline 12. & Multiple Access Interf. & $M A I$ & 0 \\
\hline 13. & Dynamic range & $P_{D R}$ & $80 \mathrm{dBm}$ \\
\hline 14. & Min. power excursion step & stepres & $.5 \mathrm{dBm}$ \\
\hline 15. & Power excursion mode & \multicolumn{2}{|c|}{ Adaptive step mode } \\
\hline 16. & SIR estimation window size & $T_{p}$ & $\begin{array}{c}20 \text { OFDM } \\
\text { Symbols }\end{array}$ \\
\hline 17. & Number of multi-paths & \multicolumn{2}{|c|}{4} \\
\hline 18. & Relative delays [ns] & \multicolumn{2}{|c|}{$[0,230,400,500]$} \\
\hline 19. & Relative Powers [dB] & \multicolumn{2}{|c|}{$[0,-9.7,-19.2,-22.8]$} \\
\hline
\end{tabular}

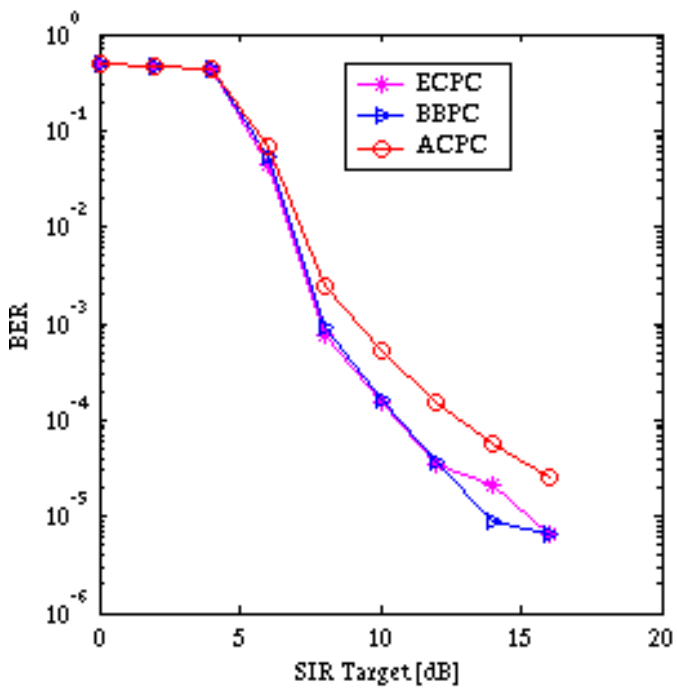

Figure 4. Performance of BBPC, ECPC and ACPC vs SIRtg at $F_{d} T_{p}=0.0598$

Furthermore, to have an insight into the behavior of BBPC as function of bandwidth occupied by one band of sub-carriers
$\left(\Delta f^{*} K_{M C}\right)$; BER performance was studied while changing the number of sub-carriers per control band while keeping the channel fading rate, coherence bandwidth and SIR target at constant values of $.0598,1.8882 \mathrm{MHz}$ and $10 \mathrm{~dB}$ respectively. The results [Fig. 6] show that as we increase the band size from one carrier per band (ECPC) to thirty-two carriers per band (BBPC), there is a negligible loss in BER performance. However, this loss increases abruptly when $\Delta f^{*} K_{M C}$ is increased beyond the coherence bandwidth of the channel $(1.8022 \mathrm{MHz})$ that reinforces the very concept of BBPC.

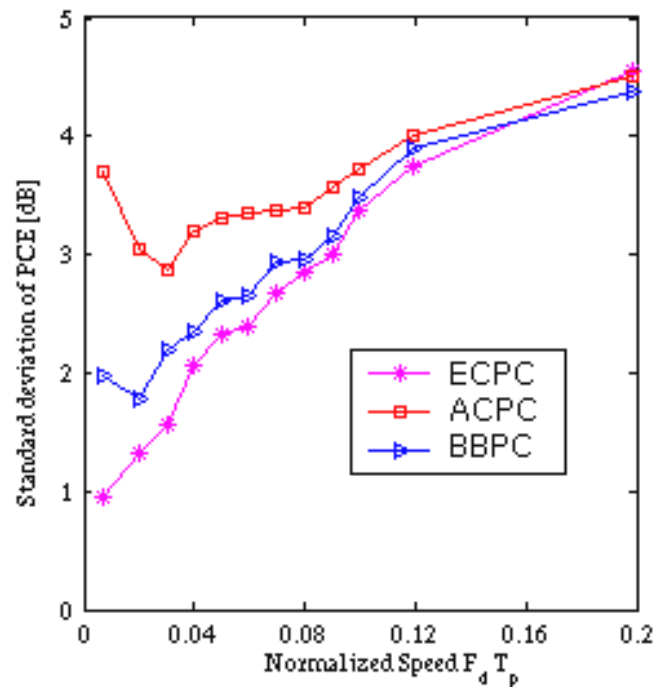

Figure 5. Performance of BBPC, ECPC and ACPC vs $F_{d} T_{p}$ at $\mathrm{SIR}_{\mathrm{tg}}=10 \mathrm{~dB}$.

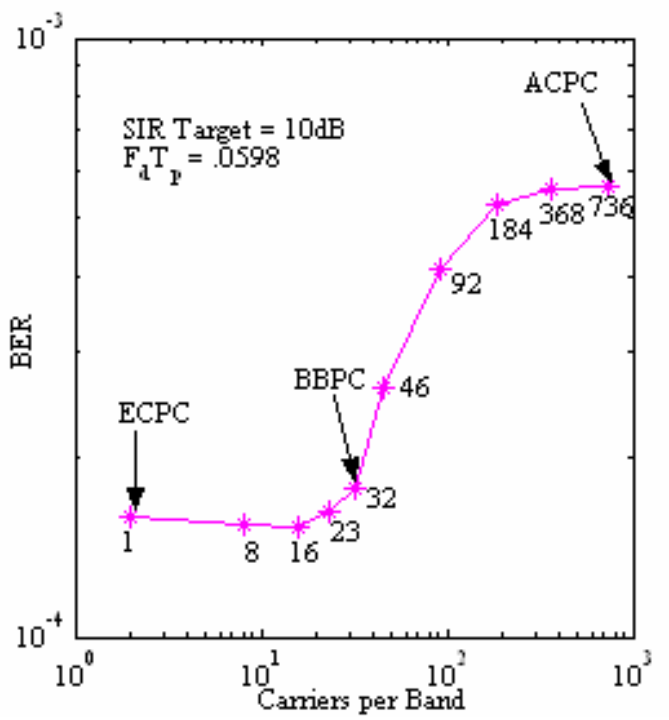

Figure 6. BER vs carriers per band at $F_{d} T_{p}=0.0598, \mathrm{SIR}_{\mathrm{tg}}=10 \mathrm{~dB}$

Since BBPC expected to perform well subjected to (5), therefore we have conducted a series of simulations to study the behavior of BBPC under channels with different delay spreads. The channels [Table III] for this experimentation are chosen to cover whole range of coherence bandwidths ranging from $\left[B_{c}>K_{M C} \Delta f\right]$ to $\left[B_{c}>K_{M C} \Delta f\right]$. In this study, BER 
performance of the system with and without BBPC in the absence of any frequency interleaving as a function of channel coherence bandwidth was studied and corresponding gains in performance are plotted in Fig.7. The performance gains is in terms of SNR, in the sense that with the same BER performance power control scheme requires that amount of less SNR. The results shows considerable BBPC performance improvement in terms of the achieved performance gains when the channel bandwidth is above $1.8022 \mathrm{MHz}$. However a performance gain of $4.15 \mathrm{~dB}$ can be seen even at $.5418 \mathrm{MHz}$, which is considerably below the bandwidth occupied by one band of sub-carriers $(1.8022 \mathrm{MHz})$. This gain is actually associated with decreased (as compared to case without BBPC) level of correlation of the fading envelop on different subcarriers within the one band due to inefficient power control where BBPC starts acting as a means of frequency inter-leaver (indirectly) rather than a true TPC mechanism.

TABLE III. SIGNALLING OVERHEAD ANALYSIS OF BBPC

\begin{tabular}{|l|c|c|c|}
\hline No & $\begin{array}{c}\text { Channel } \\
\text { Designator }\end{array}$ & $\begin{array}{c}\text { Channel delay profile }^{*} \\
{[\mathbf{n s}]}\end{array}$ & $\begin{array}{c}\text { Coherence } \\
\text { Bandwidth [MHz] }\end{array}$ \\
\hline 1 & Ch1 & {$[0,100,150,200]$} & 4.5295 \\
\hline 2 & Ch2 & {$[0,110,190,410]$} & 3.4603 \\
\hline 3 & Ch3 & {$[0,230,400,500]$} & 1.8882 \\
\hline 4 & Ch4 & {$[0,400,700,1000]$} & 1.0581 \\
\hline 5 & Ch5 & {$[0,700,1000,1500]$} & 0.6456 \\
\hline 6 & Ch6 & {$[0,805,1400,1700]$} & 0.5418 \\
\hline
\end{tabular}

* Channel power profile used is $[0,-9.7,-19.2,-22.8] \mathrm{dB}$

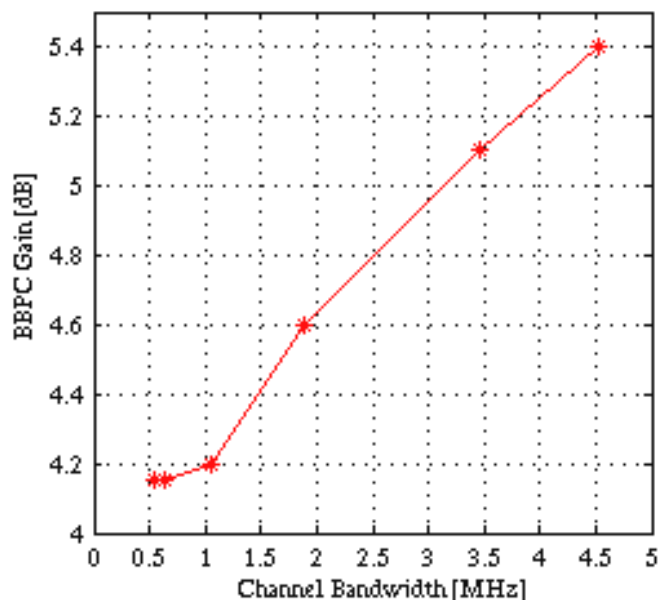

Figure 7. Performance gain in terms of required average received SNR using BBPC compared with no power control and frequency interleaving sceinario

Lastly, the feedback bandwidth required by BBPC for transmission of TPC commands on downlink has been analyzed in Table IV. It is revealed that BBPC causes only $17.5 \%$ of signaling overhead when used in adaptive step mode with a TPC command resolution of seven bits per command which reduces to only $2.5 \%$ when used in fixed step mode i.e. one bit per TPC command.
TABLE IV. SIGNALLING OVERHEAD ANALYSIS OF BBPC

\begin{tabular}{|l|l|c|c|}
\hline No & \multicolumn{1}{|c|}{ Parameter } & Symbol & Value \\
\hline 1 & Power dynamic range & $\mathrm{P}_{\mathrm{DR}}$ & $60 \mathrm{~dB}$ \\
\hline 2 & Min. power excursion step & stepres & $.5 \mathrm{~dB}$ \\
\hline 3 & TPC command resolution [bits/comd] & $\eta$ & 7 \\
\hline 4 & Signaling overhead [adaptive step mode] & $\eta^{*} \mathrm{P} / \mathrm{I}_{\mathrm{b}}$ & $17.5 \%$ \\
\hline 5 & Signaling Overhead [fixed step mode] & $\mathrm{P} / \mathrm{I}_{\mathrm{b}}$ & $2.5 \%$ \\
\hline
\end{tabular}

\section{CONCLUSIONS}

In this paper we have proposed and investigated Band Based Power Control as an uplink power control technique for MC-CDMA radio interface. It has been demonstrated through simulations that with no considerable performance loss in terms of BER and a nominal performance loss of $.27 \mathrm{~dB}$ in terms of Power Control Error, BBPC can reduce the signaling overhead to just $17.5 \%$ and $2.5 \%$ with seven bit and one bit TPC command resolution respectively when compared with ECPC which poses impractically high overheads of $80 \%$ even in fixed step mode. Secondly by employing the power control index (SIR) estimator after de-spreading and Combining, BBPC successfully overcomes the inability of ECPC to be used as an uplink power control for MC-CDMA radio interface. A gain of at least $4.6 \mathrm{~dB}$ in terms of required average SNR is achieved with BBPC compared with no power control technique.

\section{REFERENCES}

[1] S. P. W. Jarot and M. Nakagawa, " Transmission power control techniques for the reverse link of OFDM-DS-CDMA system," in Proc. IEEE International Symposium on Computers and Communications, pp.331- 337, July 1999

[2] R. Parsad, and S. Hara, "Overview of Multicarrier CDMA", Proceedings of IEEE Fourth International Symposium on Spread Spectrum Techniques \& Applications, pp.107-114, Mainz, September $22-25,1996$.

[3] Jean-Paul Linnartz "Multicarrier CDMA in an indoor wireless radio channel", IEICE Transactions on Communications, vol. E77-B, pp.900904, July 1994.

[4] Jong-Hyune Kim,"Power control and adaptive data transmission for SC/MC systems" Ph.D Thesis, August 2001, Texas A\&M university, College station.

[5] T. Nishiyama, T.shirai and M.Itami "A study on controlling Transmision power of carriers of OFDM signal combined with data symbol spreading in frequency domain", IEICE Vol.E86-A, No.8, August 2003

[6] Dejan M. Novakovic "Evaluation of power control techniques", IEEE Comm. Surveys. Fourth Qrt. 2000

[7] R. Legouable, D. Callonnec and M. Helard "Synchronization and power control processes for uplink multicarrier systems based on MC-CDMA technique" MCSS, Oberpfaffenhofen (Germany), September, 2003

[8] P. Matthias, U. Killat and F, Laue "On the statistical properties of deterministic simulation models for mobile fading channels" IEEE Vehicular Tech. Conference, Feb, 1998.

[9] X. Gui and T.S Ng "Asynchronous orthogonal multi-carrier CDMA using equal gain combining in multi-path rayleigh fading channel" ICCT 98, Beijing China

[10] S. Gunaratne, T.G Jeans, R. Tafazoli, B.G Evans "Comparison of SIR estimation for CLPC in W-CDMA Systems" IEE, 3G Mobile Comm. Technologies, March 2001. 